\section{How hosts control worms}

Nematodes are a major cause of disease and death in humans, domestic animals and wildlife. Understanding why some individuals suffer severely whereas others exposed to the same infection remain healthy may assist in the development of rational and sustainable strategies to control infection. Here, using a quantitative genetic analysis of the parasitic nematode population that had accumulated naturally in lambs, we find no apparent influence of host genetics on nematode numbers but an extremely strong influence on average worm length and fecundity. Our results indicate that in growing lambs the main manifestation of genetic resistance is the control of worm fecundity.

The lambs were all straight-bred Scottish blackface sheep from a commercial farm in southwest Strathclyde. We took faecal samples from the rectum of each lamb in late May when the lambs were 3-5 weeks old and then at four-week intervals. After taking each sample, animals were treated with a broad-spectrum anthelmintic (albendazole sulphoxide; Rycoben, Youngs Animal Health, Leyland, UK), given at the recommended dose rate of $5 \mathrm{mg}$ per $\mathrm{kg}$ body mass. We assessed the worm load after death at 6.5 months; the number of lambs studied was 501 between 1992 and 1995. We used standard parasitological methods to estimate egg counts and worm burdens ${ }^{1}$. Larval culture and post-mortem analyses indicated that more than $80 \%$ of the parasites present were Ostertagia circumcincta. At least 25 randomly chosen female $O$. circumcincta were measured for each sheep by image analysis (Foster-Findlay Associates Ltd).

The distributions of the numbers of $O$. circumcincta adults and larvae were positively skewed and we transformed the data by taking the logarithm of each trait plus one. We estimated host heritabilities and genetic correlations by residual maximum likelihood fitting an animal model with all known pedigree relationships ${ }^{2}$. We calculated standard errors from the second derivative of the log likelihood profile around the parameter estimate.

The host heritability estimates were 0 for the number of fourth-stage larvae, $0.08 \pm$ 0.09 for the number of fifth-stage larvae (sexually immature adults) and $0.14 \pm 0.10$ for the number of adult worms. There is no evidence in these results that genetic variation in the natural host influences the worm burden. In contrast, the heritability of worm length was remarkably high at $0.62 \pm 0.20$. This high heritability indicates that genetic variation in the host accounted for almost twice as much of the variation in mean

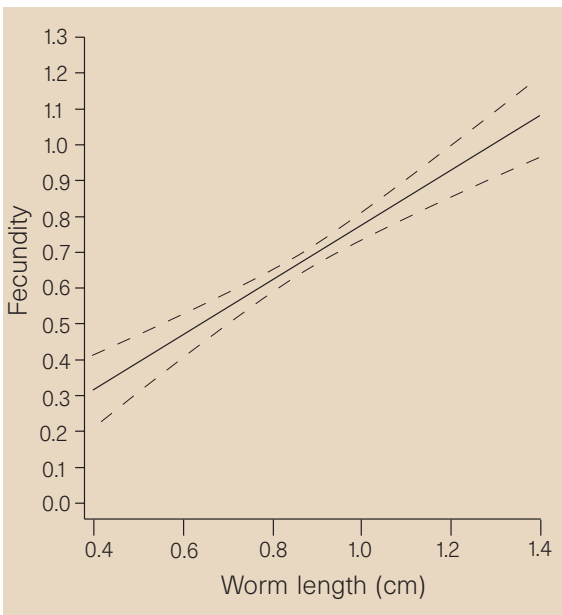

Figure 1 Relationship between worm fecundity and mean worm length. Fecundity was estimated from the log-transformed faecal egg count at slaughter divided by the log-transformed adult worm burden. The 95\% confidence limits are shown.

worm length as all other factors combined. There was a strong phenotypic correlation between the number of eggs in the worm uterus and worm length $(r=0.7$; $P<0.0001)$ and the heritability of the number of eggs in utero was also very high at 0.55 \pm 0.19 . An increase in mean worm length is associated with an increase in worm fecundity (Fig. 1). The heritability of faecal egg count at 6 months of age was $0.33 \pm 0.14$.

Our results indicate that in a flock of grazing lambs, the genetically resistant hosts ${ }^{3}$ reduce nematode egg production by decreasing worm fecundity. Although variation in egg output will be influenced by variation in worm burden and worm fecundity, there is no indication that variation in worm burden is under genetic control, at least in lambs.

We find little genetic variation in the number of fourth-stage larvae and suggest that arresting larval development is not an important mechanism of resistance to natural infection in lambs. Previous trials ${ }^{4-6}$ with deliberate infections have indicated that the main immunological mechanism that regulates worm length and fecundity of $O$. circumcincta is the quantity and specificity of parasite-specific immunoglobulin A (IgA). The quantity and specificity of IgA may therefore be an important means of identifying resistant and susceptible lambs.

M. J. Stear, K. Bairden, J. L. Duncan

P. H. Holmes, Q. A. McKellar, M. Park S. Strain, M. Murray

Glasgow University Veterinary School,

Bearsden Road, Bearsden, Glasgow G61 1QH, UK S. C. Bishop

Division of Biometrical Genetics, Roslin Institute, Roslin, Midlothian EH25 9PS, UK

\section{G. Gettinby}

Department of Statistics and Modelling Science,

University of Strathclyde, Livingston Tower,

26 Richmond Street, Glasgow G1 1XH, UK

e-mail:gvma05@udcf.gla.ac.uk
1. Armour, J., Jarrett, W. F. H. \& Jennings, F. W. Am. J. Vet. Res. 27, 1267-1278 (1966).

2. Meyer, K. Genet. Selec. Evol. 21, 317-330 (1989).

3. Bishop, S. C., Bairden, K., McKellar, Q. A., Park, M. \& Stear, M. J. Anim. Sci. 63, 423-428 (1996).

4. Stear, M. J. et al. Parasite Immunol. 17, 643-652 (1995).

5. McCririe, L. et al. Parasite Immunol. 19, 235-242 (1997).

6. Stear, M. J., Park, M. \& Bishop, S. C. Parasitol. Today 12, 438-441 (1996)

\section{Salamander with a ballistic tongue}

Lungless salamanders of the family Plethodontidae capture prey using the most enhanced tongue-protrusion mechanisms found in amphibians ${ }^{1}$. Salamanders of the genus Hydromantes are the most extreme specialists $^{1-3}$, possessing tongues that can be shot with great accuracy at prey several centimetres away (Fig. 1), reaching the target in a few milliseconds. We have found that the tongue of Hydromantes is a true projectile. It is fired from the mouth by a ballistic mechanism, and is not simply an extrapolation of the general tongue-protrusion mechanism ${ }^{1}$. The tongue reaches the prey under its own momentum.

Species of Hydromantes are found in California, Italy and France. They are the only plethodontids, the largest family of salamanders, found in the Old World, all other members being restricted to the Americas ${ }^{2,4-6}$. As well as the extremely long tongue, Hydromantes has other remarkable features including large webbed feet for climbing vertical cave walls and highly sensitive binocular vision which permits detection of prey in as low a light level as $10^{-5} \mathrm{~cd} \mathrm{~m}^{-2}$, the illumination level of an overcast, moonless night ${ }^{3}$.

Like all terrestrial salamanders, Hydromantes captures prey on a sticky tongue pad at the anterior tip of a folding tongue, supported by a skeleton of flexible, interlinked cartilages $^{1}$ (Figs 1,2). This skeleton is part of the hyobranchial apparatus, a derivative of the visceral skeleton or gill arches of ancestral vertebrates. The tongue skeleton is driven forwards by protractor muscles acting on its posterior ends, and folds to the midline as it slides forwards (Fig. 2).

In most groups of salamanders, the tongue skeleton is restricted to the floor of the mouth when at rest, but that of Hydromantes (and other members of the tribe Bolitoglossini) has tapered and elongated posterior elements that extend over the shoulders and are sheathed for their entire length in circumferential fibres of the tongue protractor muscles ${ }^{1}$ (Fig. 2a, b). The protractor muscles are visible as bulges along the sides of the body (Figs 1, 2a) and are unusual in that they exert not only pulling forces as in most salamanders, but also squeezing forces on the elongate elements of the tongue skeleton that launch the tongue from the mouth 


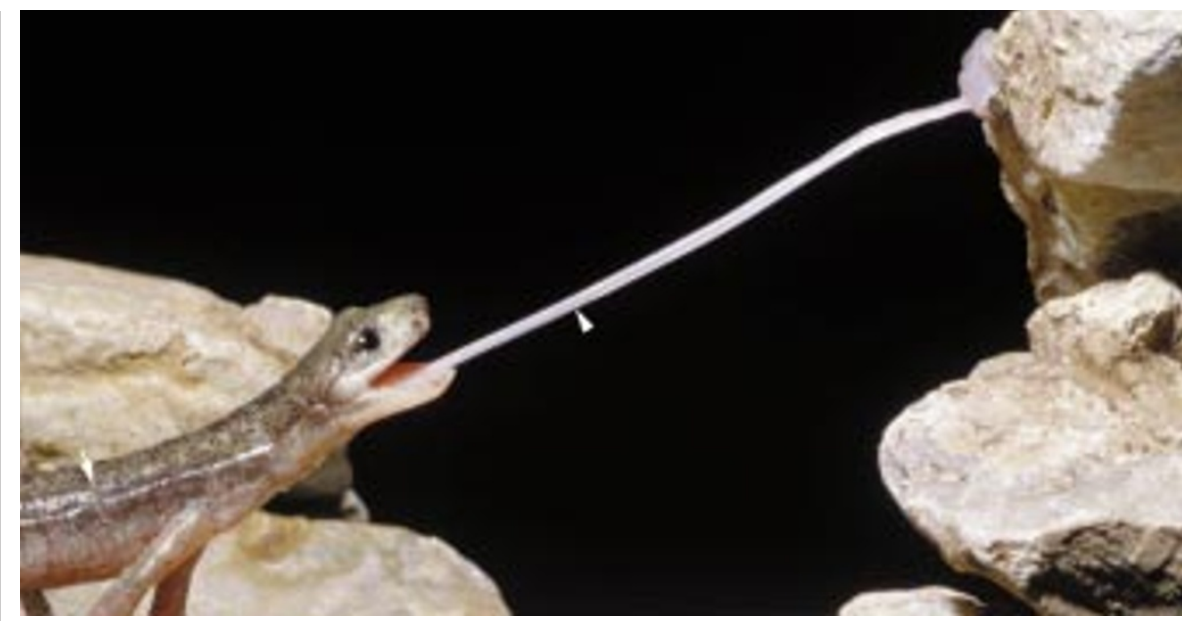

Figure 1 Live $H$. supramontis firing its tongue at a housefly. The tongue pad almost engulfs the fly yet is not fully extended. Arrow indicates the posterior extent of the tongue protractor muscle, which is visible as a bulge beneath the skin. Arrowhead points to the posterior ends of the tongue skeleton, which extends forwards to the tongue pad. It has left the mouth entirely and is anchored by the retractor muscles.

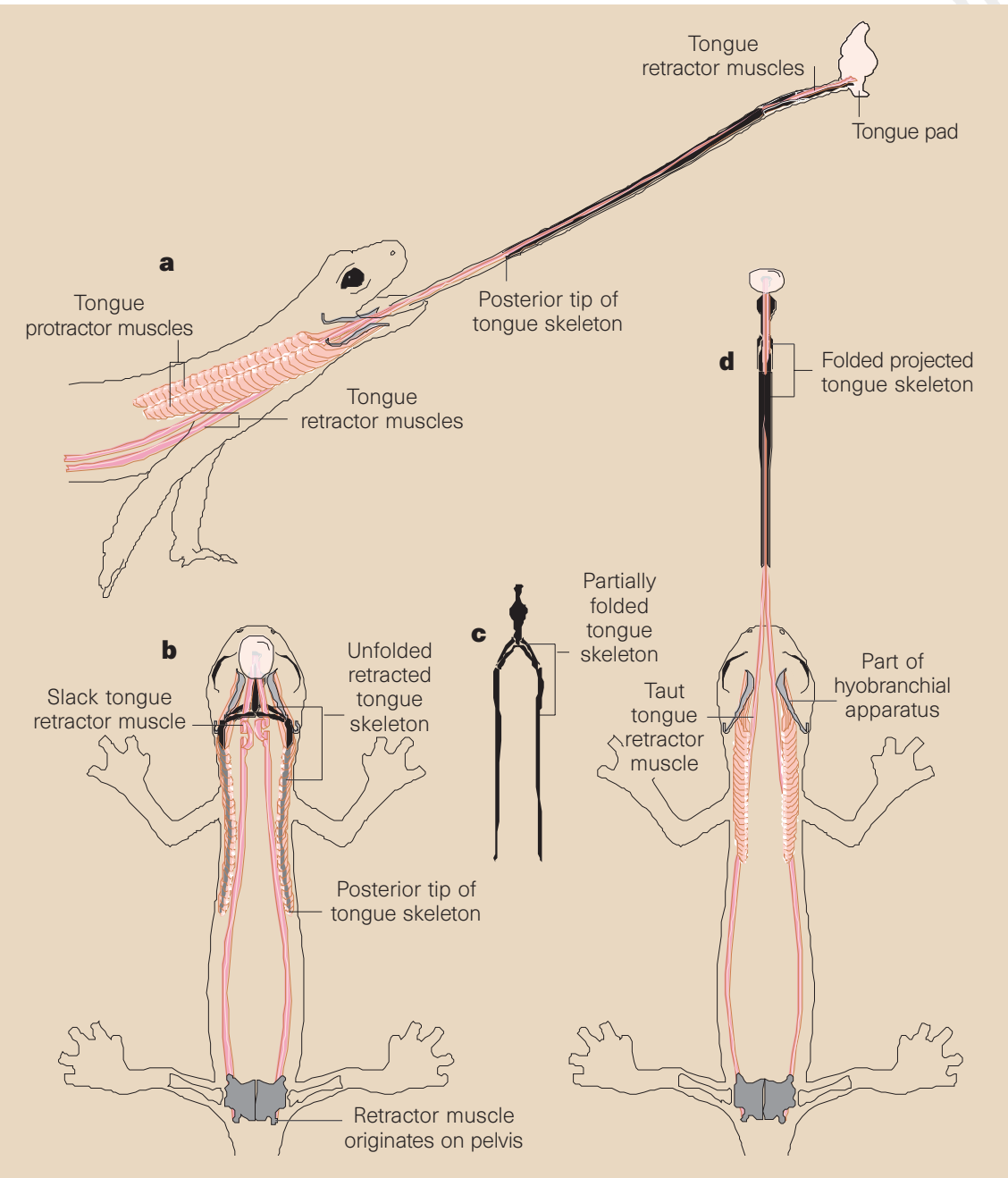

Figure 2 Tongue projection system showing tongue skeleton, associated skeletal elements and the main tongue protractor and retractor muscles. a, Lateral view corresponding to Fig. 1 showing the tongue skeleton (black) projected completely from the mouth, and bilaterally paired tongue protractor (peach) and retractor (pink) muscles. b, Dorsal view with tongue skeleton in the unfolded retracted position, elongate posterior elements (dark grey) sheathed in protractor muscles, and slack, looped retractor muscles originating on the pelvis (medium grey). c, Tongue skeleton partially folded as in an early stage of projection. $\mathbf{d}$, Dorsal view showing the tongue skeleton projected entirely from the body, beyond the contracted protractor muscles which are anchored anteriorly to the portion of the hyobranchial apparatus (light grey) that remains in the mouth. The retractor muscles, now taut, run the full length of the extended tongue and body. (much as a melon seed can be shot from between the fingers).

After the prey is struck, the tongue is returned rapidly to the mouth by lengthy retractor muscles, which in plethodontids originate on the posterior edge of the pelvis and travel uninterrupted into the tongue pad $^{1}$ (Fig. 2b, d). The retractor muscles are especially long in Hydromantes and other bolitoglossine plethodontids, to accommodate the long-distance protraction. The extra length of muscle is stored as a loop in the throat, just in front of the heart, when the tongue is at rest (Fig. 2b).

We measured preserved museum specimens of Hydromantes and found that the length of the folded tongue skeleton is less than $55 \%$ of body length. Using a handtriggered $35 \mathrm{~mm}$ camera with strobe, we obtained photographic evidence (Fig. 1) that the tongue of the Sardinian Hydromantes supramontis is protruded up to $80 \%$ of body length and that the tongue skeleton leaves the mouth completely. The tongue skeleton is projected well beyond the range within which the protractor muscles can impart force on it (Fig. 2a, d), so tongue protraction is truly ballistic.

Ballistic tongue protraction is known in chameleons ${ }^{7}$ and some frogs ${ }^{8}$, but was previously unknown in salamanders. In frogs with ballistic protraction, the tongue has no skeleton $^{8}$ and only soft tissues are projected from the mouth. Only chameleons possess longer projectile tongues than Hydromantes, however in chameleons the mechanism is reversed: the hyobranchial apparatus remains in the mouth and the tongue protractor muscle is propelled at the prey ${ }^{7}$. Hydromantes launches its entire tongue skeleton at prey and thus is the only vertebrate known to shoot part of its visceral skeleton completely out of its body as a projectile.

\section{Stephen M. Deban}

\section{David B. Wake}

Museum of Vertebrate Zoology and Department of Integrative Biology,

3101 Valley Life Sciences Building,

University of California, Berkeley,

California 94720-3160, USA

email:deban@socrates.berkeley.edu

\section{Gerhard Roth}

Brain Research Institute,

University of Bremen,

FB 2, D-28334 Bremen, Germany

1. Lombard, R. E. \& Wake, D. B. J. Morphol. 153, 39-80 (1977).

2. Dunn, E. R. The Salamanders of the Family Plethodontidae (Smith College, Northampton, 1926).

3. Roth, G. Visual Behavior in Salamanders (Springer, Berlin, 1987).

4. Lanza, B., Caputo, V., Nascetti, G. \& Bullini, L. Morphologic and Genetic Studies of the European Plethodontid Salamanders:

Taxonomic Inferences (Genus Hydromantes) (Museo Regionale di Scienze Naturali, Torino, 1995).

5. Frost, D. R. Amphibian Species of the World (Allen, Lawrence, 1985).

6. Wake, D. B. Mem. S. Cal. Acad. Sci. 4, 1-111 (1966).

7. Wainwright, P. C. \& Bennett, A. F. J. Exp. Biol. 168, 23-40 (1992).

8. Nishikawa, K. C. \& Gans, C. J. Exp. Biol. 199, 2511-2529 (1996). 